\title{
DESENVOLVIMENTO DE SOBREMESA LÁCTEA TIPO FROZEN YOGURT COM CARACTERÍSTICAS FUNCIONAIS
}

\author{
R. S. JULIANO ${ }^{1}$, S. S. J. de SARKIS ${ }^{1}$, A. C. PINHEIRO ${ }^{1}$, A. C. FEAR ${ }^{1}$, C. A. ZAMBELLI ${ }^{1}$, M. \\ M. AUGUSTO ${ }^{1}$ \\ ${ }^{1}$ Universidade Federal do Rio Grande, Escola de Química e Alimentos \\ E-mail para contato: dqmmarta@furg.br
}

\begin{abstract}
RESUMO - As sobremesas congeladas são constituídas de ingredientes lácteos e nãolácteos em proporções adequadas, que incorporada de ar produz uma textura suave e cremosa. Formulações de sobremesa láctea foram desenvolvidas utilizando como base láctea proporções de leitelho:leite $(3: 1,1: 1,1: 3)$, com e sem a presença de emulsificante. A composição das formulações foram quantificadas quanto aos teores de proteína, extrato seco total, gordura, resíduo mineral fixo, lactose, acidez titulável e $\mathrm{pH}$. Também foram determinados o overrun e a dureza. As formulações desenvolvidas apresentaram $78 \%$ de base láctea. Foi observado que os fatores experimentais e suas interações influenciaram significativamente $(\mathrm{p}<0,05)$ o overrun e a dureza. As medidas de overrun variaram de 88,9 à $144,4 \%$. A formulação F3 (1:3; com emulsificante) apresentou maior overrun e dureza. Já a formulação F2 (3:1; sem emulsificante) obteve menor valor de overrun. Todas as formulações independente da proporção leitelho:leite, elaboradas com a presença de emulsificante, apresentaram maior rendimento e dureza.
\end{abstract}

\section{INTRODUÇÃO}

O desenvolvimento de novos produtos é um desafio para a indústria alimentícia, à medida que procura atender à demanda dos consumidores por alimentos saudáveis e atrativos (Komatsu et al., 2008). Nessa categoria estão as sobremesas lácteas que constituídas de ingredientes lácteos e não-lácteos em variedade e proporções adequadas, congeladas ou não, aeradas e cremosas, como por exemplo, os sorvetes, mousses, flans, dentre outros.

Sorvetes ou gelados comestíveis são produtos alimentícios obtidos a partir de uma emulsão de gorduras e proteínas, com ou sem adição de outros ingredientes e submetidas ao congelamento (Silva et al., 2012). O frozen yogurt é uma sobremesa fermentada e congelada que associa o valor nutricional do iogurte com o sabor refrescante do sorvete, possui baixo teor de gordura e lactose (Goff, 2011). Nesse sentido, o frozen yogurt pode ser considerado uma alternativa saudável ao sorvete por sua composição nutricional e seu conteúdo em proteínas, cálcio e vitaminas (Pereira et al., 2012).

O leitelho ou buttermilk é obtido a partir do batimento do creme durante a produção de manteiga. No processo ocorre a ruptura da membrana que protege o glóbulo de gordura do leite, liberando os lipídios apolares, e os componentes constituintes da membrana (Malin et al., 1994). O uso do buttermilk em sistemas alimentícios está relacionado com a sua composição, em especial os 
componentes da emulsão, tais como os fosfolípidos originários da membrana do glóbulo de gordura do leite (MFGM) que têm potencial para aplicações funcionais e nutracêuticas (O’Connell; Fox, 2000).

O interesse por produtos alimentícios saudáveis e nutritivos tem crescido mundialmente, o que resulta em um desafio para a indústria láctea. Estudos têm dado ênfase ao valor nutricional dos ingredientes lácteos, como uma alternativa promissora para a indústria de alimentos. Nesse sentido, o objetivo do trabalho foi o desenvolvimento de sobremesa láctea do tipo frozen yogurt, utilizando diferentes proporções de leitelho e leite na composição da base láctea.

\section{MATERIAL E MÉTODOS}

\subsection{Matérias-Primas e Ingredientes}

As matérias-primas utilizadas para a elaboração da sobremesa láctea tipo frozen yogurt foram o leite pasteurizado e o leitelho fornecido pela empresa Danby Cosulati. Os ingredientes utilizados na formulação foram: leite em pó desnatado (Elegê), sacarose (açúcar refinado União), glicose líquida(Yoki), creme de leite com $48 \%$ de gordura (Santa Clara), gelatina em pó sem sabor (Dr. Oetker), polpa de morango (MOR 501®) fornecido pela empresa Borsato, fermento lácteo(YOY $\left.{ }^{\circledR}\right)$ da empresa Coalhopar. Também foram utillizados como aditivos: estabilizante super liga neutra (Duas Rodas), emulsificante (Emulstab) e aromatizante artificial de morango (Duas Rodas).

\subsection{Desenvolvimento das Formulações}

A composição da formulação teste foi de acordo com Corte (2008), utilizando as mesmas proporções para glicose, creme e polpa de morango. Para as demais formulações a composição da sobremesa foi modificada em base a um planejamento experimental.

Para cada formulação de sobremesa foi conduzido um experimento com a mistura de leitelho e de leite pasteurizado em base ao planejamento experimental, totalizando seis formulações nas proporções 3:1, 1:3, 1:1 respectivamente. Na preparação da sobremesa láctea, a mistura leitelho/leite foi pré- aquecida até $50^{\circ} \mathrm{C}$, adicionada de leite em pó, creme de leite e açúcar. A seguir foi homogeneizada, pasteurizada à $80^{\circ} \mathrm{C}$ durante 30 minutos e, após, resfriada à $45^{\circ} \mathrm{C}$. Após, a mistura foi dividida em volumes iguais para elaboração da bebida láctea e do mix. A bebida foi preparada por inoculação do fermento lácteo e encubada em banho termostatizado a $45^{\circ} \mathrm{C}$ até $\mathrm{pH} 4,6$, seguida de resfriamento à $12^{\circ} \mathrm{C}$.

O mix foi elaborado por acréscimo de gelatina, estabilizante e glicose, homogeneizado, pasteurizado à temperatura de $65^{\circ} \mathrm{C}$ durante 30 minutos. Após, resfriado e levado à maturação a 4 ${ }^{\circ} \mathrm{C}$ durante 4 horas. Finda esta etapa, foi realizado o batimento da calda maturada e da bebida láctea, em sorveteira durante 30 minutos à $-6{ }^{\circ} \mathrm{C}$. A sobremesa láctea tipo frozen yogurt foi acondicionada em recipientes plásticos com capacidade para $2 \mathrm{~L}$, armazenada à $-18{ }^{\circ} \mathrm{C}$ e mantida nessa temperatura até a realização das análises físico-químicas. 


\subsection{Caracterização do Leite e Leitelho}

As matérias primas foram analisadas quanto aos teores de proteína, gordura, extrato seco total, resíduo mineral fixo, lactose, acidez titulável e $\mathrm{pH}$ segundo a Instrução Normativa $\mathrm{n}^{\circ} 68$ de 12 de dezembro de 2006 (Brasil, 2006).

\subsection{Caracterização da Sobremesa Láctea Tipo Frozen Yogurt}

A sobremesa elaborada foi quantificada quanto aos teores de proteína, extrato seco total, gordura, resíduo mineral fixo (RMF), lactose, $\mathrm{pH}$ e acidez titulável de acordo com os métodos recomendados pela Association of Oficial Analytical Chemists (1995).

\subsection{Overrun}

O rendimento da sobremesa tipo frozen yogurt foi determinado pelo método proposto por Innocente et al. (2002) e calculado de acordo com a Equação 1.

$$
\text { overrun }=\frac{\text { (volume do sorvete) }- \text { (volume da mistura) }}{\text { volume da mistura }} * 100 \%
$$

\subsection{Análise Instrumental da Textura}

Para cada formulação foram preparados 3 recipientes com capacidade de $200 \mathrm{ml}$, preenchidos com as amostras, fechados e armazenados a $-18^{\circ} \mathrm{C}$. Para as análises, as amostras e um probe cilíndrico de acrílico com diâmetro de $12 \mathrm{~mm}$ foram mantidos a $-10^{\circ} \mathrm{C}$ por 24 horas de antecedência para a determinação da textura. A dureza foi avaliada utilizando um texturômetro modelo TAXT Plus e s condições de análise foram uma célula de compressão de $100 \mathrm{~N}$ com velocidade de penetração de $50 \mathrm{~mm} / \mathrm{min}$ e profundidade de $18 \mathrm{~mm}$.

\subsection{Planejamento Experimental}

No estudo foi realizado um planejamento do tipo misturado, onde os fatores foram: a proporção leitelho e leite em três níveis $(3: 1 ; 1: 1$ e 1:3) e presença ou ausência de emulsificante. O planejamento $3 \times 2$ resultou em 6 experimentos que em duplicata totalizaram 12 experimentos. Todas as análises físico-químicas do leite, leitelho e produto final foram realizadas em triplicata. $\mathrm{Na}$ Tabela 1 podem ser visualizados os níveis das variáveis reais e codificadas da matriz experimental.

Tabela 1:Matriz experimental das variáveis reais e codificadas do planejamento experimental

\begin{tabular}{lcccc}
\hline \multirow{2}{*}{ Formulação } & \multicolumn{2}{c}{ Variáveis Codificadas } & \multicolumn{2}{c}{ Variáveis Reais } \\
\cline { 2 - 5 } & Proporção (leitelho: leite) & $\begin{array}{c}\text { Presença de } \\
\text { emulsificante }\left(\mathrm{X}_{2}\right)\end{array}$ & $\begin{array}{c}\text { Proporção de } \\
\text { leitelho:leite }\end{array}$ & Emulsificante \\
\hline F1 & -1 & 1 & $3: 1$ & Presente \\
F2 & -1 & -1 & $3: 1$ & Ausente \\
F3 & 1 & 1 & $1: 3$ & Presente \\
F4 & 1 & -1 & $1: 3$ & Ausente \\
F5 & 0 & -1 & $1: 1$ & Ausente \\
F6 & 0 & 1 & $1: 1$ & Presente \\
\hline
\end{tabular}


Os resultados obtidos foram analisados por análise de variância para verificar diferenças significativas entre os níveis dos fatores e estudo de interação entre eles e teste de Tukey com nível de $5 \%$ de significância.

\section{RESULTADOS E DISCUSSÃO}

Na Tabela 2 pode ser observado o conteúdo das formulações testes para a elaboração da sobremesa láctea tipo frozen yogurt até a definição da formulação final.

Tabela 2: Formulações teste para o desenvolvimento da sobremesa tipo frozen yogurt

\begin{tabular}{lcccc}
\hline \multirow{2}{*}{ COMPOSIÇÃO } & \multicolumn{5}{c}{ FORMULAÇÕES } \\
\cline { 2 - 5 } & I & II & III & IV \\
\hline Base Láctea & 70 & 68,8 & 71 & 69 \\
(leitelho:leite) $(\% \mathrm{v} / \mathrm{v})$ & & 7 & 12 & 8 \\
Sacarose (\%p/v) & 7 & 4 & 7 & 5 \\
Glicose (\%p/v) & 4 & 3 & 3 & 3 \\
Creme (\%p/v) & 3 & 1 & 1 & 1 \\
Liga Neutra (\%p/v) & 1 & 4 & 4 & 4 \\
Polpa (\%p/v) & 4 & 1 & - & 1 \\
Leite em Pó (\%p/v) & 7 & 2,7 & 1 & 1 \\
Estabilizante (\%p/v) & 1 & 1,5 & - & - \\
Cultura lática (\%p/v) & 1 & & - & \\
Aromatizante $(\% \mathrm{p} / \mathrm{v})$ & 2 & 7 & & \\
\hline
\end{tabular}

O aromatizante adicionado nas formulações I e II foi substituído por gelatina que proporcionou melhoria na estabilidade da sobremesa. A formulação final do produto pode ser visualizada na Tabela 3.

Tabela 3: Formulação final da sobremesa láctea tipo frozen yogurt

\begin{tabular}{ll}
\hline Composição & Percentagem $(\mathbf{p} / \mathbf{p})$ \\
\hline Leite em pó & $6,0 \%$ \\
Creme de leite & $1,5 \%$ \\
Sacarose & $6,0 \%$ \\
Estabilizante & $1,0 \%$ \\
Gelatina & $0,5 \%$ \\
Glicose & 3,0 \\
Polpa & $4,0 \%$ \\
Base láctea (leitelho:leite) & $78,0 \%$ \\
\hline
\end{tabular}

$\mathrm{Na}$ tabela 4, podem ser visualizados os valores médios da composição físicoquímica do leite e do leitelho utilizados para a elaboração da sobremesa láctea. 
Tabela 4:Caracterização físico-química do leite e do leitelho utilizados na elaboração da sobremesa láctea tipo frozen yogurt

\begin{tabular}{|c|c|c|}
\hline Determinações & Leite & Leitelho \\
\hline Umidade (\%) & $87,12 \pm 0,43$ & $90,43 \pm 0,21$ \\
\hline Proteína1 (\%) & $3,52 \pm 0,06$ & $3,42 \pm 0,12$ \\
\hline $\operatorname{Gordura}(\%)$ & $3,00 \pm 0,01$ & $1,30 \pm 0,01$ \\
\hline $\operatorname{RMF}^{2}(\%)$ & $0,69 \pm 0,01$ & $0,65 \pm 0,01$ \\
\hline $\operatorname{EST}^{3}(\%)$ & $12,80 \pm 0,43$ & $9,57 \pm 0,21$ \\
\hline Lactose (\%) & $4,62 \pm 0,04$ & $3,60 \pm 0,04$ \\
\hline $\mathrm{AT}^{4}$ (g ácido lático/ $/ 100$ I & $0,18 \pm 0,01$ & $0,09 \pm 0,03$ \\
\hline pH & $6,7 \pm 0,01$ & $6,6 \pm 0,01$ \\
\hline
\end{tabular}

Pode-se observar que os valores obtidos (Tabela 4) para a caracterização físico-quimica do leite encontram-se de acordo com os parâmetros estabelecidos pela legislação (Brasil, 2011).

A composição do leitelho encontra-se dentro da faixa estabelecida pelos autores Lubudzisz e Stepaniak (2011). Segundo Sodini et al.(2006) e Gassi et al. (2008), diferenças na composição físico-química do leitelho podem ocorrer devido à constituição do creme e as variáveis operacionais utilizadas na fabricação da manteiga.

A caracterização físico-química das sobremesas lácteas elaboradas com as diferentes proporções de leitelho e leite são apresentadas na Tabela 5.

Tabela 5: Média da composição físico-química da sobremesa láctea tipo frozen yogurt

\begin{tabular}{|c|c|c|c|c|c|c|c|}
\hline Formulação & Proteína $^{1}$ & EST $^{3}$ & $\mathbf{R M F}^{2}$ & Acidez $^{4}$ & Gordura & Lactose & pH \\
\hline F1 & $6,57^{\mathrm{a}, \mathrm{b}, \mathrm{c}} \pm 0,04$ & $30,89^{\mathrm{a}, \mathrm{b}} \pm 0,44$ & $1,39^{\mathrm{a}} \pm 0,0$ & $0,43^{\mathrm{c}} \pm 0,01$ & $2,65^{\mathrm{e}} \pm 0,0$ & $5,99^{\mathrm{c}} \pm 0,0$ & $5,8^{\mathrm{a}} \pm 0,01$ \\
\hline $\mathbf{F} 2$ & $6,52^{\mathrm{b}} \pm 0,05$ & $31,88^{\mathrm{a}} \pm 0,64$ & $1,40^{\mathrm{a}} \pm 0,0$ & $0,46^{\mathrm{d}} \pm 0,01$ & $2,50^{\mathrm{f}} \pm 0,0$ & $5,98^{\mathrm{c}} \pm 0,0$ & $5,7^{\mathrm{a}} \pm 0,01$ \\
\hline F3 & $6,61^{\mathrm{a}} \pm 0,03$ & $31,19^{\mathrm{a}, \mathrm{b}} \pm 0,36$ & $1,40^{\mathrm{a}} \pm 0,0$ & $0,62^{\mathrm{b}} \pm 0,01$ & $3,55^{\mathrm{a}} \pm 0,0$ & $6,20^{\mathrm{a}} \pm 0,0$ & $5,3^{\mathrm{b}} \pm 0,01$ \\
\hline F4 & $6,54^{\mathrm{b}, \mathrm{c}} \pm 0,03$ & $31,12^{\mathrm{a}, \mathrm{b}} \pm 0,7^{-}$ & $1,40^{\mathrm{a}} \pm 0,0$ & $0,63^{\mathrm{b}} \pm 0,01$ & $3,40^{\mathrm{b}} \pm 0,0$. & $6,11^{\mathrm{b}} \pm 0,0$ & $5,3^{\mathrm{b}} \pm 0,01$ \\
\hline F5 & $6,61^{\mathrm{a}} \pm 0,02$ & $30,79^{\mathrm{a}, \mathrm{b}} \pm 1,0($ & $1,39^{\mathrm{a}} \pm 0,0^{\prime}$ & $0,75^{\mathrm{a}} \pm 0,01$ & $3,00^{\mathrm{d}} \pm 0,0$. & $6,19^{\mathrm{a}} \pm 0,0$ & $5,2^{\mathrm{c}} \pm 0,01$ \\
\hline F6 & $6,59^{\mathrm{a}, \mathrm{c}} \pm 0,01$ & $30,44^{\mathrm{b}} \pm 0,73$ & $1,39^{\mathrm{a}} \pm 0,0^{\prime}$ & $0,74^{\mathrm{a}} \pm 0,01$ & $3,20^{\mathrm{c}} \pm 0,0$ & $6,12^{\mathrm{b}} \pm 0,0$ & $5,2^{\mathrm{c}} \pm 0,01$ \\
\hline
\end{tabular}

a,b,c... Média com subscritos iguais na mesma coluna não diferem significativamente entre $\operatorname{si}(\mathrm{p}<0,05)$;

$* \mathrm{n}=6 ;{ }^{1}$ Proteína $=\% \mathrm{NT}$ x $6.38 ;{ }^{2} \mathrm{RMF}=$ resíduo mineral fixo; ${ }^{3} \mathrm{EST}=$ extrato seco total;

${ }^{4}$ Acidez $=(\mathrm{g}$ ácido $\quad$ lático $/ 100 \mathrm{~mL})$

A proporção do leitelho e leite influenciou significativamente a composição físico-química do produto enquanto que o emulsificante os teores de proteína, gordura, acidez e $\mathrm{pH}$. A interação entre os fatores de estudo foi significativa $(\mathrm{p}<0,05)$ para a proteína, lactose, acidez e pH indicando a dependência dos fatores entre si. 
Os teores de proteína nas formulações variaram de 6,52 a $6,61 \%$, e as formulações F3 e F5 diferiram significativamente $(\mathrm{p}<0,05)$ das formulações F2 e F4. Para os sólidos totais a faixa de variação foi de 30,44 a 31,88 \%. De acordo com Goff (2011) os teores de sólidos totais para o frozen yogurt se encontram entre $30-36 \%$. Os valores de $\mathrm{pH}$ das formulações apresentaram uma variação de 5,2 a 5,8 unidades. E a acidez titulável de 0,43 a 0,44 \% de ácido láctico. Nas formulações F1 e F2 verifica-se maior pH e menor acidez. Segundo Tramontina et al. (2011) uma acidez titulável elevada e baixo valor de $\mathrm{pH}$ em um produto fermentado é decorrência da produção de ácido lático por ação de bactérias fermentativas. Os valores de lactose apresentaram variação de 5,98 a $6,2 \%$. Nas formulações F1 e F2 com maior proporção de leitelho foram encontrados menor conteúdo de lactose. O teor de lipídios apresentou valores de 2,5 a 3,5\%. Conforme Guinard et al. (1994) a gordura do leite favorece as características sensoriais, proporcionando textura macia, cremosidade e sabor, aprimorando a qualidade do produto.

O overrun é o indicador de rendimento da sobremesa, isto é, corresponde a incorporação de ar na massa durante o batimento. Quanto maior for o overrun, mais leve e suave é a sobremesa (GOFF, 2011). A Tabela 6 apresenta a média e desvio padrão do overrun e dureza das formulações do frozen yogurt.

Tabela 6: Média e desvio padrão do overrun e dureza da sobremesa láctea tipo frozen yogurt

\begin{tabular}{lll}
\hline Formulação & Overrun $(\%)$ & Dureza $(\mathbf{N})$ \\
\hline F1 & $95,7^{\mathrm{b}} \pm 4,25$ & $290,8^{\mathrm{c}} \pm 17,9$ \\
F2 & $88,9^{\mathrm{b}} \pm 0,01$ & $82,9^{\mathrm{d}} \pm 2,9$ \\
F3 & $144,4^{\mathrm{a}} \pm 22,2$ & $498,4^{\mathrm{a}} \pm 39,6$ \\
F4 & $107,7^{\mathrm{b}} \pm 3,40$ & $46,6^{\mathrm{d}} \pm 7,8$ \\
F5 & $100,0^{\mathrm{b}} \pm 0,01$ & $279,3^{\mathrm{c}} \pm 14,5$ \\
F6 & $111,1^{\mathrm{b}} \pm 0,06$ & $362,7^{\mathrm{b}} \pm 26,0$ \\
\hline${ }^{\mathrm{a}, \mathrm{b}, \mathrm{c}, \mathrm{d} .}$ Médias com subscritos iguais na mesma coluna não diferem significativamente $(\mathrm{p}<0,05)$ & $\mathrm{n}=3$
\end{tabular}

Os fatores de estudo e sua interação influenciaram significativamente $(\mathrm{p}<0,05)$ o overrun e a dureza. Observa-se na Tabela 6 que as medidas do overrun variaram de 88,9 à 144,4\%, a formulação F3 apresentou maior overrun, significando maior quantidade de ar incorporado à mistura durante o batimento e que poderá interferir na qualidade do produto final. O rendimento pode ser influenciado por diversos fatores, como por exemplo, a velocidade e temperatura de batimento, as características funcionais da mistura láctea dentre outros (Goff, 2011). Na formulação F2 com maior proporção de leitelho e ausência de emulsificante foi obtido o menor valor de overrum, visto que a presença do aditivo tem por função melhorar a incorporação e distribuição do ar no produto durante o batimento.

Na Tabela 6 pode ser observado que as formulações de sobremesa apresentaram uma variação de dureza de 46,6 a 498,4N. As amostras com emulsificante (F1, F3 e F6) obtiveram maior resistência à penetração e consequentemente maior dureza. A textura de uma sobremesa depende do estado de agregação dos glóbulos de gordura, da quantidade de ar incorporado, do tamanho e da quantidade dos cristais de gelo formados, ou seja, está relacionada com a estrutura da matriz alimentícia (KAYA e TEKIN, 2000). 


\section{CONCLUSÃO}

A composição da formulação apresentou $6,0 \%(\mathrm{p} / \mathrm{v})$ de leite em pó, $1,5 \%(\mathrm{p} / \mathrm{v})$ de creme de leite, $6,0 \%(\mathrm{p} / \mathrm{v})$ de sacarose, $1,0 \%(\mathrm{p} / \mathrm{v})$ de estabilizante, $0,5 \%(\mathrm{p} / \mathrm{v})$ de gelatina, 3,0\% (p/v) de glicose, $4,0 \%(\mathrm{p} / \mathrm{v})$ de polpa e base láctea de $78,0 \%(\mathrm{v} / \mathrm{v})$.

A caracterização físico-química das formulações foi influenciada significativamente $(p<0,05)$ pela proporção de leitelho:leite para os teores de proteína, EST, gordura, RMF, lactose, acidez e pH. A interação entre os fatores foi significativa para lactose, proteínas, acidez e pH.

Os fatores experimentais e suas interações influenciaram significativamente $(p<0,05)$ o overrun e a dureza. A formulação F3 (1:3; com emulsificante) apresentou maior overrun e dureza. Já a formulação F2 (3:1; sem emulsificante) obteve menor valor de overrun. Todas as formulações independente da proporção leitelho:leite, elaboradas com a presença de emulsificante, apresentaram maior rendimento e dureza.

\section{REFERÊNCIAS}

ASSOCIATION OF OFICIAL ANALYTICAL CHEMISTS - AOAC INTERNATIONAL. Official Methods of Analysis. 16. ed. Arlington: AOAC International v.2, 1995.

BRASIL. Ministério da Agricultura Pecuária e Abastecimento. Departamento de Inspeção de Produtos de Origem Animal. Instrução Normativa n.62 Aprova e oficializa o Regulamento Técnico de Identidade e Qualidade do Leite e Leite Pasteurizado. Diário Oficial Da União, 29 de dezembro de 2011.

BRASIL. Ministério da Agricultura, Pecuária e Abastecimento. Instrução Normativa n.68 de 12 de dezembro de 2006. Oficializa os Métodos Analíticos Oficiais Físico - Químicos, para controle de leite e produtos lácteos, em conformidade com o anexo desta Instrução Normativa, determinando que sejam utilizados nos Laboratórios Nacionais Agropecuários. Diário Oficial da União, seção1, página 8, de 14 dezembro, 2006.

CORTE, F. F.D. Desenvolvimento de frozen yogurt com propriedades funcionais Dissertação (Mestrado em Ciências e Tecnologia de Alimentos). Universidade Federal de Santa Maria, Santa Maria, 2008.

GASSI, J., FAMELART, M.; LOPEZ, C. Heat treatment of cream affects the physicochemical properties of sweet buttermilk. Dairy Science technology. v.88, p.369-385, 2008.

GOFF, H. D. Ice cream and frozen desserts: product types. In: Encyclopedia of dairy sciences, $2^{\circ}$ ed. Academic Press, London, U K, v.2 p.893-912, 2011.

GUINARD, J. X.; C. LITTLE; C. MARTY; T. R. Effect of sugar and acid on the acceptability of frozen yogurt to a student population. Journal Dairy Science. v. 77, p.1232-1238, 1994.

INNOCENTE, N.; COMPARIN, D.; CORRADINI, C. Proteose-peptone whey fraction as emulsifier in ice-cream preparation. International Dairy Journal, v. 12, p.69-74, 2002.

KAYA, S.; TEKIN, A.R.The effect of salep content on the rheological characteristics of a typical ice-cream mix. Journal of Food Engineering, v. 47, p. 59-62, 2001. 
KOMATSU, T. R.; BURITI, F. C. A.; SAAD, S. M. I. Inovação, persistência e criatividade superando barreiras no desenvolvimento de alimentos probióticos. Revista Brasileira de Ciências Farmacêuticas, v.44, n.3, jul/set., 2008.

LUBUDZISZ, Z.; STEPANIAK, L. Buttermilk. In: Encyclopedia of dairy sciences, $2^{\circ}$ ed. Academic Press, London, U K, v.2 p.489-495 , 2011.

MALIN, E.L.; BASCH, J.J.; SHIEH, J.J.; SULLIVAN, B.C.; HOLSINGER, V.H. Detection of adulteration of buttermilk powder by gel electrophoresis. Journal of Dairy Science v. 77, p. 2199-2206, 1994.

O’CONNELL, J. E.; FOX, P. F.Heat stability of buttermilk. Journal of Dairy Science v.83, p.1728-1732, 2000.

PEREIRA, G. G.; RAFAEL, L. M.; GAJO, A. A.; RAMOS, T. M., PINTO, S. M.; ABREU, L. R. de; RESENDE, J. V. de. Influência do pH nas características físico-químicas e sensoriais de frozen yogurt de morango. Ciências Agrárias, Londrina v. 33, n.2, p. 657-686, abr. 2012.

SILVA, W. M. da; SILVA, L. R. da; SILVA, A. L. da; ONE, G. M. C A visão bioquímica do sorvete. In: simpósio paraibano de saúde: tecnologia, saúde e meio-ambiente à serviço da vida, 2012, João Pessoa. Disponível em <http://www.institutobioeducacao.org.br/livrosimposiodefinitivo.pdf> acesso em: 24 de julho de 2013.

SODINI, I.; MORIN, P.; OLABI, A.; JIMÉNEZ-FLORES, R. Compositional and Functional Properties of Buttermilk: A Comparison Between Sweet, Sour, and Whey Buttermilk. Journal of Dairy Science v.89, p.525 - 536, 2006.

TRAMONTINA, T; RICHARDS N. S. P. S.; SILVA M. E. Desenvolvimento e caracterização físico-química, microbiológica e sensorial de iogurte adicionado de cereais integrais. Anais do XVIII Congresso Nacional de Laticínios [S.I]. N³21, v.56, p. 225-229, julho/agosto 2011. 